\title{
Robust Design using Desirability Function to the Combined-Array with Multiple Quality Characteristics
}

\author{
Yong-Man Kwon and Jang-Jae Lee ${ }^{\dagger}$
}

\begin{abstract}
Robust design is an approach to reducing performance variation of quality characteristic values in quality engineering. Taguchi has an idea that mean and variation are handled simultaneously to reduce the expected loss in products and processes. In the Taguchi parameter design, the product-array approach using orthogonal arrays is mainly used. However, it often requires an excessive number of experiments. An alternative approach, which is called the combined-array approach, was studied. In these studies, only single quality characteristic (or response) was considered. In this paper we propose how to simultaneously optimize for multiple quality characteristics (or multiresponse) using desirability function when we used the combined-array approach to assign control and noise factors.
\end{abstract}

Key words: Robust Design, Combined-Array, Simultaneously Optimize

\section{Introduction}

The Taguchi parameter design is an approach to reducing performance variation of response values in products and processes. Products and their manufacturing processes are influenced both by control factors that can be controlled by designers and by noise factors that are difficult or expensive to control such as environmental conditions. The basic idea of parameter design is to identify, through exploiting interactions between control factors and noise factors, appropriate settings of control factors that make the system's performance robust to changes in the noise factors. Parameter design is a quality improvement technique proposed by the Japanese quality expert Taguchi ${ }^{[1,2]}, \operatorname{Kackar}^{[3]}$, and others.

The control factors are assigned to an inner array, which is an orthogonal array. For each row in the inner array, the noise factors are assigned to an outer array, also an orthogonal array. Because the outer array is run for every row in the inner array, we call this setup a product array. A large number of experimental trials in Taguchi's product array may be required because the

Department of Computer science and statistics, Chosun University, Gwangju

${ }^{\dagger}$ Corresponding author : jijlee21@gmail.com

(Received : February 18, 2013, Revised : March 20, 2013,

Accepted : March 25, 2013) noise array is repeated for every row in the control array.

There have been efforts for integrating Taguchi's important notion of heterogeneous variability the standard experimental design and modeling technology provided by response surface methodology. They combined control and noise factors in a single design matrix, which we call a combined array.

The combined array approach was first proposed by Welch, Yu, Kang, and Sacks ${ }^{[4]}$. The initial motivation of the combined array is the run-size saving. Related approaches were discussed by Vining and Myers ${ }^{[5]}$, Box and Jones ${ }^{[6]}$, Shoemaker, Tsui, and Wu $(1991)^{[7]}$, and Myers, Khuri and Vining, etc ${ }^{[8]}$. Treatment of the mean and variance responses via a constrained optimization was discussed by Vining and Myers ${ }^{[5]}$.

In many experimental situations, a number of responses are measured for a given setting of design variables. Khuri and Conlon ${ }^{[9]}$ introduced a procedure for the simultaneous optimization of multiple responses using a distance function.

\section{Estimated Mean and Variance Models in Multiresponse System}

Suppose the response $y$, depends on control variables (or factors) and noise variables. Let a set of control var- 
iables be denoted by $\underline{x}=\left(x_{1}, x_{2}, \ldots, x_{l}\right)^{\prime}$ and and a set of noise variables by $\underline{z}=\left(z_{1}, z_{2}, \ldots, z_{m}\right)^{\prime}$. Suppose that all response functions in a multiresponse system depend on the same set of $\underline{x}$ and $\underline{z}$ that they can be represented by second order models within a certain region of interest. Let $N$ be the number of experimental runs and $r$ be the number of response functions. The $i$ th second order model is

$$
\begin{aligned}
y_{i}(\underline{x}, \underline{z}) & =\beta_{i 0}+\underline{x}^{\prime} \underline{\beta}_{i}+\underline{x}^{\prime} \beta_{i} \underline{x}+\underline{z}^{\prime} R_{i} \underline{z}+\underline{z}^{\prime} \underline{\gamma}_{i}+\underline{z}^{\prime} D_{i} \underline{x}+\varepsilon_{i} \\
i & =1,2, \ldots, r
\end{aligned}
$$

Where $\underline{\beta}_{i}$ is $l \times 1, \not_{i}$ is $m \times 1 B_{i}^{\prime}=B_{i}$ is $l \times l, R_{i}^{\prime}=R_{i}$ is $m \times m, D_{i}$ is $l \times m$, which are vectors or matrices of unknown regression parameters, and $\varepsilon_{i}$ is a random error associated with the $i$ th response.

Equation (1) can be expressed in matrix notation as

$$
\underline{y}_{i}=X \underline{\theta}_{i}+\underline{\varepsilon}_{i} \quad i=1,2, \ldots, r
$$

in which $\underline{y}_{i}$ is an $N \times 1$ vector of observations on the $i$ th response, $X$ is an $N \times p$ full column rank matrix of known constants, $\underline{\theta}_{i}$ is the $p \times 1$ column vector of unknown regression parameters, and $\underline{\varepsilon}_{i}$ is a vector of random errors associated with the $i$ th response. We also assume that

$$
\begin{aligned}
E\left(\underline{\varepsilon}_{i}\right) & =\underline{0}, \operatorname{Var}\left(\underline{\varepsilon}_{i}\right)=\sigma_{i i} I_{N}, \operatorname{Cov}\left(\underline{\varepsilon}_{i}, \underline{\varepsilon}_{j}\right)=\sigma_{i j} I_{N} \\
i, j & =1,2, \ldots, r, \quad i \neq j
\end{aligned}
$$

From (2) and (3), ordinary least squares(OLS) estimation is the same as generalized least squares(GLS) estimation ${ }^{[10]}$. Thus, the fitted $i$ th second-order model can be written as

$$
\begin{aligned}
\hat{y}_{i}(\underline{x}, \underline{z}) & =b_{i 0}+\underline{x}^{\prime} \underline{b}_{i}+\underline{x}^{\prime} B_{i} \underline{x}+\underline{z}^{\prime} \hat{R}_{i} \underline{z}+\underline{z}^{\prime} \underline{r}_{i}+z^{\prime} \hat{D}_{i} \underline{x} \\
i & =1,2, \ldots, r
\end{aligned}
$$

The noise variables $\underline{z}$ are not controllable and they are random variables. In the absence of other knowledge, $\underline{z}$ would be usually uniformly distributed over $R_{z}(-1 \leq z \leq 1)$.

Box and Jones ${ }^{[6]}$ modeled the mean and variance separately in a single response. But, we are interested in showing the estimated mean and variance response models in multiple responses.

Let $\hat{m}_{i}(\underline{x})$ ith estimated mean response at an $\underline{x}$ averaged over the noise variables

$$
\hat{m}_{i}(\underline{x})=\int_{R_{z}} \hat{y}_{i}(\underline{x}, \underline{z}) p(\underline{z}) d_{z}, \quad i=1,2, \ldots, r
$$

where $p(\underline{z})$ is a probability density function of $\underline{z}$, and $\underline{z}$ has a uniform distribution over $R_{z}$. Box and Jones ${ }^{[6]}$ showed that the $i$ th estimated mean becomes

$$
\hat{m}_{i}(\underline{x})=b_{i 0}+\underline{x}^{\prime} \underline{b}_{i}+\underline{x}^{\prime} \hat{B}_{i} \underline{x}+\frac{1}{3} \operatorname{tr} \hat{R}_{i}, \quad i=1,2, \ldots, r
$$

where $\operatorname{tr} \hat{R}_{i}$ is the trace of the matrix $\hat{R}_{i}$. Let us write $\hat{v}_{i}(x)$ for the $i$ th mean square variation about the $i$ th mean response

$$
\hat{v}_{i}(\underline{x})=\int_{R_{z}}\left(\hat{y}_{i}(\underline{x}, \underline{z})-\hat{m}_{i}(\underline{x})\right)^{2} p(\underline{z}) d_{z}, \quad i=1,2, \ldots, r
$$

Let us call this measure the $i$ th estimated variance, which becomes

$$
\hat{v}_{i}(\underline{x})=\frac{1}{3}\left(\underline{r}_{i}+\hat{D}_{i} \underline{x}\right)^{\prime}\left(\underline{r}_{i}+\hat{D}_{i} \underline{x}\right)+\hat{A}_{i}, \quad i=1,2, \ldots, r
$$

where $\hat{A}_{i}=\left[4 \sum_{j=1}^{m}\left(r_{j j}^{i}\right)^{2}+5 \sum_{j=1}^{m-1} \sum_{k=j+1}^{m}\left(r_{j k}^{i}\right)^{2}\right] / 45$ and $r_{j k}^{i}$ is the $j$ th row and $k$ th column element of the matrix $\hat{R}_{i}$.

\section{Simultaneous Optimization for Multiple Quality Characteristics}

\subsection{Simultaneous Optimization for Multiple Mean} Responses

Derringer and Suich ${ }^{[11]}$ introduced a simultaneous optimization method of multiple responses based on the concept of utility or desirability. We can combine the simultaneous optimization of their work with the notion of Taguchi's work. We propose a new simultaneous optimization method for a set of estimated mean responses using the desirability function.

The desirability function involves transformation of each estimated mean response $\hat{m}_{i}(\underline{x})$ to a desirability value $d_{i}(\underline{x})$, where $\left(0 \leq d_{i}(\underline{x}) \leq 1\right)$. The value of $d_{i}(\underline{x})$ increases as the "desirability" of the corresponding response increases. The individual desirabilities are then combined using the geometric mean

$$
D_{m}(\underline{x})=\left(d_{i}(\underline{x}) \times d_{2}(\underline{x}) \times \ldots \times d_{r}(\underline{x})\right)^{1 / r}
$$

Thus, we propose a new simultaneous-optimization method for the estimated mean responses using the desirability function as

$$
\max _{\underline{x} \in R_{x}} D_{m}(\underline{x})=\max _{\underline{x} \in R_{x}}\left(d_{i}(\underline{x}) \times d_{2}(\underline{x}) \times \ldots \times d_{r}(\underline{x})\right)^{1 / r}
$$

where $R_{x}$ is the region of interest on a set of control variables $x$. 
All the quality characteristics(or responses) are classified into three basic situations, namely, the larger-isbetter characteristics, the smaller-is-better characteristics and the nominal-is-best characteristics. The estimated mean responses are classified into three basic situations the same as all quality characteristics. Thus, we propose three class of a desirability function $d_{i}(\underline{x})$.

\subsubsection{Desirability Function for Larger-is-better Quality Characteristics}

For the larger-is-better case, $d_{i}(\underline{x})$ increases as $\hat{m}_{i}(x)$ increases and is employed where $\hat{m}_{i}(x)$ is to be maximized. We shall consider the transformations given by

$$
d_{i}(\underline{x})=\left\{\begin{array}{cl}
0 & \hat{m}_{i}(\underline{x}) \leq m_{i^{*}} \\
{\left[\frac{\hat{m}_{i}(\underline{x})-m_{i^{*}}}{m_{i}^{*}-m_{i^{*}}}\right]^{p}} & m_{i^{*}} \leq \hat{m}_{i}(\underline{x}) \leq m_{i}^{*} \\
1 & m_{i}^{*} \leq \hat{m}_{i}(\underline{x})
\end{array}\right.
$$

Where $m_{i^{*}}$ is the minimum acceptable value of $\hat{m}_{i}(\underline{x})$, and the value $m_{i^{*}}$ is the maximum (or target) value of $\hat{m}_{i}(\underline{x})$ over the region of interest $R_{x}$. A large value of $p$ would be specified if it were very desirable for the value of $\hat{m}_{i}(\underline{x})$ to increase rapidly above $m_{i^{*}}$. On the other hand, a small value of $p$ would be specified if having values of $\hat{m}_{i}(\underline{x})$ considerably above $m_{i^{*}}$ were not of critical importance. Fig. 1 shows the plot of the desirability function for the larger-is-better quality characteristics, Equation (8).

\subsubsection{Desirability Function for Smaller-is-better \\ Quality Characteristics}

For the smaller-is-better case, $d_{i}(\underline{x})$ increases as $\hat{m}_{i}(\underline{x})$ decreases and is employed where $\hat{m}_{i}(\underline{x})$ is to be minimized. We shall consider the transformations given by

$$
d_{i}(\underline{x})=\left\{\begin{array}{cl}
0 & m_{i}^{*} \leq \hat{m}_{i}(\underline{x}) \\
{\left[\frac{m_{i^{*}}-\hat{m}_{i}(\underline{x})}{m_{i}^{*}-m_{i^{*}}}\right]^{q}} & m_{i^{*}} \leq \hat{m}_{i}(\underline{x}) \leq m_{i}^{*} \\
1 & \hat{m}_{i}(\underline{x}) \leq m_{i^{*}}
\end{array}\right.
$$

where $m_{i^{*}}$ is the minimum (or target) value of $\hat{m}_{i}(\underline{x})$ over the region of interest $R_{x}$, and $m_{i^{*}}$ is the maximum acceptable value of $\hat{m}_{i}(\underline{x})$. A large value of $q$ would be specified if it were very desirable for the value of $\hat{m}_{i}(\underline{x})$ to decrease rapidly above $m_{i^{*}}$. On the other hand, a small value of $q$ would be specified if having values of $\hat{m}_{i}(\underline{x})$ considerably above $m_{i^{*}}$ were not of critical importance. Fig. 2 shows the plot of the desirability function for the smaller-is-better quality characteristics, Equation (9).

\subsubsection{Desirability Function for Nominal-is-best} Quality Characteristics

For the nominal-is-best case, we shall consider the transformations given by

$$
d_{i}(\underline{x})= \begin{cases}{\left[\frac{\hat{m}_{i}(\underline{x})-m_{i^{*}}}{\tau_{i}-m_{i^{*}}}\right]^{s}} & m_{i^{*}} \leq \hat{m}_{i}(\underline{x}) \leq \tau_{i} \\ {\left[\frac{\hat{m}_{i}(\underline{x})-m_{i^{*}}}{\tau_{i}-m_{i^{*}}}\right]^{t}} & \tau_{i} \leq \hat{m}_{i}(\underline{x}) \leq m_{i}^{*} \\ 1 & m_{i}^{*} \leq \hat{m}_{i}(\underline{x}) \text { or } \quad \hat{m}_{i}(\underline{x}) \geq m_{i}^{*}\end{cases}
$$

where $m_{i^{*}}$ is the minimum acceptable value of $\hat{m}_{i}(\underline{x})$ and $m_{i^{*}}$ is the maximum acceptable value of $\hat{m}_{i}(\underline{x})$. Values of $\hat{m}_{i}(\underline{x})$ outside these limits would make the entire product unacceptable. The value $\tau_{i}$ is the most-desirable (or target) value of $\hat{m}_{i}(\underline{x})$. The values of $s$ and $t$ play the same role as $p$ and $q$ in Equations (8) and (9). Fig. 3 shows the plot of the desirability function for the smaller-is-better quality characteristics, Equation (10).

\subsection{Simultaneous Optimization of Multiple Variance Responses}

Variation (or variance) for all the quality characteristics is the smaller-is-better quality characteristics because Variance of quality characteristics is the smaller-is-better case. Thus, the $i$ th estimated variance is the smaller-is-better case. We propose a new simultaneous optimization method for a set of estimated variance responses using the desirability function.

The desirability function involves transformation of each estimated variance response $\hat{v}_{i}(\underline{x})$ to a desirability value $d_{i}^{*}(\underline{x})$, where $\left(0 \leq d_{i}^{*}(\underline{x}) \leq 1\right)$. The value of $d_{i}^{*}(\underline{x})$ increases as the "desirability" of the corresponding response increases. The individual desirabilities are then combined using the geometric mean

$$
D_{v}(\underline{x})=\left(\dot{d}_{1}^{*}(\underline{x}) \times d_{2}^{*}(\underline{x}) \times \ldots \times d_{r}^{*}(\underline{x})\right)^{1 / r}
$$

Thus, we propose a new simultaneous-optimization method for the estimated variance responses using the desirability function as

$$
\max _{\underline{x} \in R_{x}} D_{v}(\underline{x})=\max _{\underline{x} \in R_{x}}\left(d_{i}^{*}(\underline{x}) \times d_{2}^{*}(\underline{x}) \times \ldots \times d_{r}^{*}(\underline{x})\right)^{1 / r}
$$




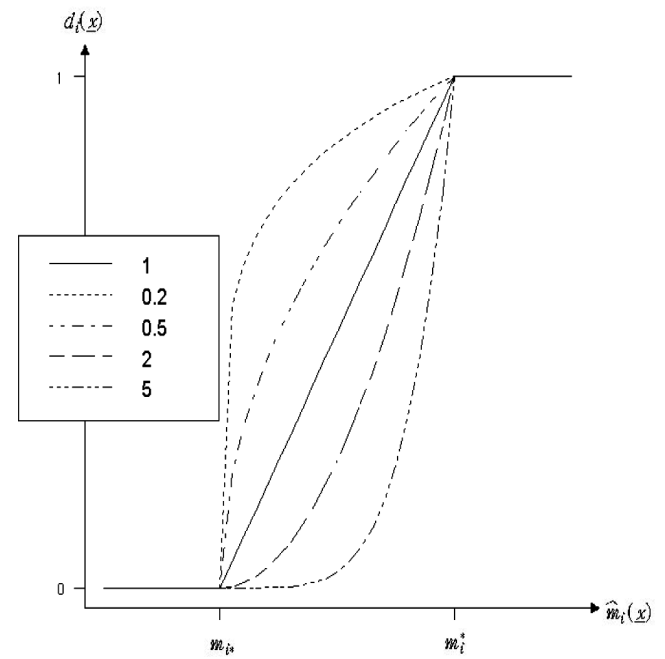

Fig. 1. Larger-is-better case

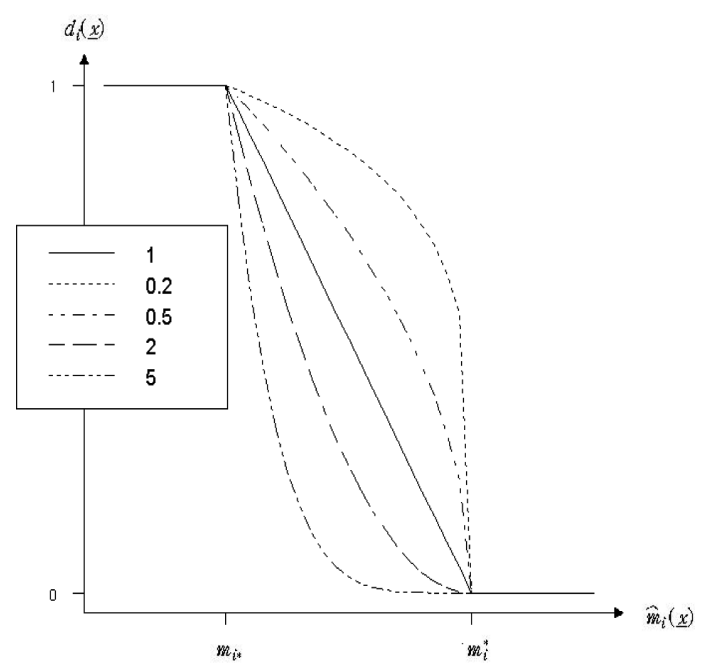

Fig. 2. Smaller-is-better case

where $R_{x}$ is the region of interest on a set of control variables $\underline{x}$. Because the $i$ th estimated variance is the smaller-is-better case, $d_{i}(\underline{x})$ increases as $\hat{v}_{i}(\underline{x})$ decreases and is employed where $\hat{v}_{i}(\underline{x})$ is to be minimized. We shall consider the transformations given by

$$
d_{i}(\underline{x})=\left\{\begin{array}{cl}
0 & v_{i}^{*} \leq \hat{v}_{i}(\underline{x}) \\
{\left[\frac{v_{i}^{*}-\hat{v}_{i}(\underline{x})}{v_{i}^{*}-v_{i^{*}}}\right]^{q}} & v_{i^{*}} \leq \hat{v}_{i}(\underline{x}) \leq v_{i}^{*} \\
1 & \hat{v}_{i}(\underline{x}) \leq v_{i^{*}}
\end{array}\right.
$$

where $v_{i^{*}}$ is the minimum (or target) value of $\hat{v}_{i}(\underline{x})$ over

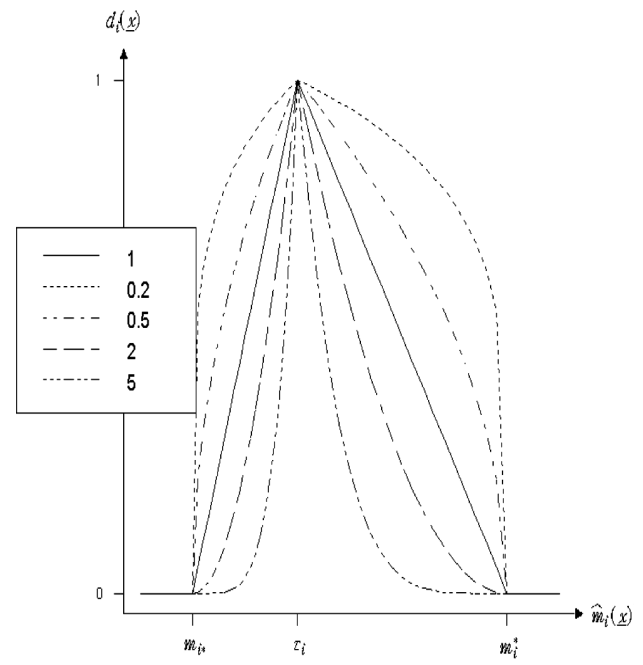

Fig. 3. Nominal-is-best case

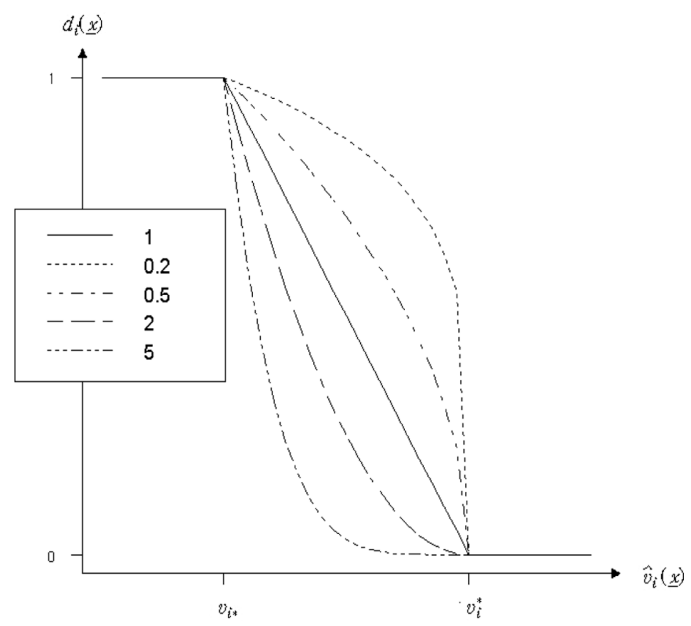

Fig. 4. Variance case

the region of interest $R_{x}$, and $v_{i}^{*}$ is the maximum acceptable value of $\hat{v}_{i}(\underline{x})$. Figure 4 shows the plot of the desirability function for a set of estimated variance responses, Equation (12).

\subsection{Proposed Simultaneous-optimization Measu-} rement

Let us find conditions on a set of control variables $\underline{x}$ which optimize simultaneously for a set of estimated mean responses and estimated variance responses. If all estimated mean and all the estimated variance attain their individual optimum values at the same set $\underline{x}$ of operating conditions, then the problem of simultaneous 
optimization is obviously solved. This ideal optimum rarely occurs. In more general situations we might consider finding compromising conditions on the control variables that are somewhat favorable to all mean responses and all the estimated variance. Such deviation of the compromising conditions from the ideal optimum condition can be formulated by means of the desirability function.

We propose a simultaneous optimization for a set of estimated mean responses and estimated variance responses over the region of interest $R_{x}$ using proposed $D_{m}(\underline{x})$ and $D_{v}(\underline{x})$. From Equations (7) and (11), the proposed simultaneous-optimization measure can be written as

$$
p_{d}=\max _{\underline{x} \in R_{x}} P_{d}(\underline{x})=\max _{\underline{x} \in R_{x}}\left[\lambda D_{m}(\underline{x})+(1-\lambda) D_{v}(\underline{x})\right]
$$

where $0 \leq \lambda \leq 1$. This is a criterion in which $D_{m}(\underline{x})$ and $D_{v}(\underline{x})$ take on different degrees of importance.

If a simultaneous optimum value is much different from its corresponding individual optimum value, we may choose a bound on it and then reoptimize $P_{d}$. Also we may analyze $P_{d}$ sequentially as the acceptable values for $\lambda$ is varied.

\section{Numerical Example}

In this section we give a numerical example, consisting of a multiresponse system of three response variables, $y_{1}, y_{2}$ and $y_{3}$, and three control variables, $x_{1}$ (or $A$ ), $x_{2}$ (or $B$ ) and $x_{3}$ (or $C$ ), and one noise variable $z$ (or $N$ ) which are assigned in the orthogonal array, $L_{18}\left(2^{1} \times 3^{7}\right)$. Suppose that the objective is to find the simultaneous optimum conditions for increasing the strength of plastic product $y_{1}$, reducing the wear on the plastic product $y_{2}$ and equalling the specific length of plastic product $y_{3}$. Also suppose there is a noise factor $N$ with three levels $\left(N_{0}\right.$ : good condition, $N_{1}$ : normal condition, $N_{2}$ : bad condition). The control factors are listed in Table 1. Table 2 gives a set of strength data $y_{1}$, wear data $y_{2}$ and length data $y_{3}$.

Table 1. Factors and levels

\begin{tabular}{lccc}
\hline \multicolumn{1}{c}{ Control factor } & -1 level & 0 level & 1 level \\
\hline$A:$ time $(\min )$ & 120 & 125 & 130 \\
$B:$ temperature $\left({ }^{\circ} \mathrm{C}\right)$ & 60 & 70 & 80 \\
$C:$ stir speed $(\mathrm{rpm})$ & 300 & 350 & 400 \\
\hline
\end{tabular}

Table 2. Assignment of sources and data in the combined array

\begin{tabular}{|c|c|c|c|c|}
\hline & e $A B C$ e e e $Z$ & $y_{1}$ & $y_{2}$ & $y_{3}$ \\
\hline 1 & $\begin{array}{cccccccc}-1 & -1 & -1 & -1 & -1 & -1 & -1 & -1\end{array}$ & 222 & 32 & 174 \\
\hline 2 & $\begin{array}{lllllllll}-1 & -1 & 0 & 0 & 0 & 0 & 0 & 0\end{array}$ & 181 & 25 & 170 \\
\hline 3 & $\begin{array}{lllllllll}-1 & -1 & 1 & 1 & 1 & 1 & 1 & 1\end{array}$ & 262 & 28 & 164 \\
\hline 4 & 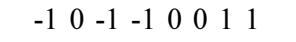 & 187 & 31 & 88 \\
\hline 5 & $\begin{array}{llllllll}-1 & 0 & 0 & 0 & 1 & 1 & -1 & -1\end{array}$ & 222 & 22 & 103 \\
\hline 6 & $\begin{array}{llllllll}-1 & 0 & 1 & 1 & -1 & -1 & 0 & 0\end{array}$ & 303 & 28 & 92 \\
\hline 7 & $\begin{array}{llllllll}-1 & 1 & -1 & 0 & -1 & 1 & 0 & 1\end{array}$ & 211 & 16 & 206 \\
\hline 8 & $\begin{array}{lllllllll}-1 & 1 & 0 & 1 & 0 & -1 & 1 & -1\end{array}$ & 190 & 27 & 185 \\
\hline 9 & $\begin{array}{lllllllllllll}-1 & 1 & 1 & -1 & 1 & 0 & -1 & 0\end{array}$ & 290 & 21 & 169 \\
\hline 10 & $\begin{array}{llllllll}1 & -1 & -1 & 1 & 1 & 0 & 0 & -1\end{array}$ & 195 & 30 & 126 \\
\hline 11 & $\begin{array}{lllllllll}1 & -1 & 0 & -1 & -1 & 1 & 1 & 0\end{array}$ & 212 & 30 & 139 \\
\hline 12 & $\begin{array}{llllllll}1 & -1 & 1 & 0 & 0 & -1 & -1 & 1\end{array}$ & 296 & 19 & 142 \\
\hline 13 & $\begin{array}{lllllllll}1 & 0 & -1 & 0 & 1 & -1 & 1 & 0\end{array}$ & 193 & 19 & 115 \\
\hline 14 & $\begin{array}{lllllllll}1 & 0 & 0 & 1 & -1 & 0 & -1 & 1\end{array}$ & 168 & 31 & 83 \\
\hline 15 & $\begin{array}{llllllll}1 & 0 & 1 & -1 & 0 & 1 & 0 & -1\end{array}$ & 312 & 27 & 112 \\
\hline 16 & $\begin{array}{lllllllll}1 & 1 & -1 & 1 & 0 & 1 & -1 & 0\end{array}$ & 210 & 35 & 181 \\
\hline 17 & $\begin{array}{llllllll}1 & 1 & 0 & -1 & 1 & -1 & 0 & 1\end{array}$ & 165 & 29 & 202 \\
\hline 18 & $\begin{array}{lllllllll}1 & 1 & 1 & 0 & -1 & 0 & 1 & -1\end{array}$ & 317 & 26 & 228 \\
\hline
\end{tabular}

Each of the three responses was fitted to a second order regression model from the Table 1 using Equation (4). The estimated response models by the method of least squares are given by

$$
\begin{aligned}
\hat{y}_{1}(\underline{x}, \underline{z})= & 194.42-0.30 x_{1}+59.18 x_{2}+2.18 x_{3}-2.28 x_{1}^{2} \\
& +65.63 x_{2}^{2}-12.73 x_{3}^{2}-3.33 x_{1} x_{2}+18.68 x_{1} x_{3} \\
& -1.32 x_{2} x_{3}-10.75 z+13.76 x_{1} z-0.85 x_{2} z \\
& -3.18 x_{3} z+23.33 z^{2} \\
\hat{y}_{2}(\underline{x}, \underline{z})= & 20.61-1.55 x_{1}-1.45 x_{2}+1.33 x_{3}+1.35 x_{1}^{2} \\
& -1.21 x_{2}^{2}+8.28 x_{3}^{2}+1.24 x_{1} x_{2}+1.02 x_{1} x_{3} \\
& +0.42 x_{2} x_{3}-0.06 z-0.81 x_{1} z-1.90 x_{2} z \\
& +0.52 x_{3} z+0.32 z^{2} \\
& \\
\hat{y}_{3}(\underline{x}, \underline{z})= & 102.36+24.45 x_{1}-5.54 x_{2}-4.55 x_{3}+86.84 x_{1}^{2} \\
& -3.54 x_{2}^{2}-13.26 x_{3}^{2}-7.90 x_{1} x_{2}-11.77 x_{1} x_{3} \\
& +16.53 x_{2} x_{3}-14.61 z-1.33 x_{1} z-8.72 x_{2} z \\
& +13.38 x_{3} z-0.32 z^{2}
\end{aligned}
$$

From Equation (14) through (16), using the mean and variance response Equation (5) and (6), the estimated mean and variance response models are given by 
Table 3. Simultaneous optimization for $P_{d}$

\begin{tabular}{cc|ccc|c|ccccccc}
\hline \multicolumn{1}{c}{} & \multicolumn{1}{c}{} & \multicolumn{3}{c|}{ Mean } & \multicolumn{3}{c}{ Variance } \\
\hline$\lambda$ & $1-\lambda$ & $x_{1}$ & $x_{2}$ & $x_{3}$ & $P_{d}$ & $d_{1}$ & $d_{2}$ & $d_{3}$ & $d_{1}^{*}$ & $d_{2}^{*}$ & $d_{3}^{*}$ \\
\hline 0.0 & 1.0 & -1.00 & 1.00 & -0.37 & 0.49 & 0.98 & 0.83 & 0.98 & 0.72 & 0.17 & 1.00 \\
0.1 & 0.9 & -1.00 & 1.00 & -0.37 & 0.54 & 0.98 & 0.83 & 0.98 & 0.72 & 0.17 & 1.00 \\
0.2 & 0.8 & -1.00 & 1.00 & -0.37 & 0.58 & 0.98 & 0.83 & 0.98 & 0.72 & 0.17 & 1.00 \\
0.3 & 0.7 & -1.00 & 1.00 & -0.37 & 0.62 & 0.98 & 0.83 & 0.98 & 0.72 & 0.17 & 1.00 \\
0.4 & 0.6 & -1.00 & 1.00 & -0.36 & 0.67 & 0.98 & 0.83 & 0.99 & 0.72 & 0.17 & 0.99 \\
0.5 & 0.5 & -1.00 & 1.00 & -0.36 & 0.71 & 0.98 & 0.83 & 0.99 & 0.72 & 0.17 & 0.99 \\
0.6 & 0.4 & -1.00 & 1.00 & -0.33 & 0.76 & 0.98 & 0.84 & 1.00 & 0.73 & 0.16 & 0.96 \\
0.7 & 0.3 & -1.00 & 1.00 & -0.33 & 0.80 & 0.98 & 0.84 & 1.00 & 0.73 & 0.16 & 0.96 \\
0.8 & 0.2 & -0.98 & 1.00 & -0.23 & 0.85 & 0.97 & 0.87 & 1.00 & 0.73 & 0.15 & 0.87 \\
0.9 & 0.1 & -0.96 & 1.00 & -0.12 & 0.90 & 0.96 & 0.89 & 1.00 & 0.73 & 0.14 & 0.77 \\
1.0 & 0.0 & -1.00 & -1.00 & 1.00 & 0.72 & 0.56 & 1.00 & 0.68 & 0.01 & 0.01 & 0.00 \\
\hline
\end{tabular}

$\hat{m}_{1}(\underline{x})=-0.30 x_{1}+59.18 x_{2}+2.18 x-2.28 x_{1}^{2}+65.63 x_{2}^{2}-$ $12.73 x_{3}^{2}-3.33 x_{1} x_{2}+18.68 x_{1} x_{3}-1.32 x_{2} x_{3}+195.23$

$\hat{m}_{2}(\underline{x})=-1.55 x_{1}-1.45 x_{2}+1.33 x_{3}+1.35 x_{1}^{2}-1.21 x_{2}^{2}+8.28 x_{3}^{2}$ $+1.24 x_{1} x_{2}+1.02 x_{1} x_{3}+0.42 x_{2} x_{3}+20.72$

$\hat{m}_{3}(\underline{x})=24.45 x_{1}-5.54 x_{2}-4.55 x_{3}+86.84 x_{1}^{2}-3.54 x_{2}^{2}-$ $13.26 x_{3}^{2}-7.90 x_{1} x_{2}-11.77 x_{1} x_{3}+16.53 x_{2} x_{3}+102.25$

$\hat{v}_{1}(\underline{x})=\left(2.67 x_{1}-10.79 x_{2}-0.55 x_{3}+112.22\right)^{2} / 3+48.36$

$\hat{v}_{2}(\underline{x})=\left(-0.81 x_{1}-1.90 x_{2}+0.52 x_{3}-0.06\right)^{2} / 3+0.01$

$\hat{v}_{3}(\underline{x})=\left(-1.33 x_{1}-8.72 x_{2}+13.38 x_{3}-14.61\right)^{2} / 3+0.01$

The region of interest $R_{x}$ is given by the inequality $-1 \leq x_{1}, x_{2} \leq 1$. The ranges for $\hat{m}_{1}(\underline{x}), \hat{m}_{2}(\underline{x}), \hat{m}_{3}(\underline{x}), \hat{v}_{1}(\underline{x})$, $\hat{v}_{2}(\underline{x})$, and $\hat{v}_{3}(\underline{x})$ over $R_{x}$ are respectively,

$$
\begin{aligned}
& m_{1^{*}}=145.393 \leq \hat{m}_{1}(\underline{x}) \leq m_{1}^{*}=331.042 \\
& m_{2^{*}}=17.943 \leq \hat{m}_{2}(\underline{x}) \leq m_{2}^{*}=33.490 \\
& m_{3^{*}}=70.131 \leq \hat{m}_{3}(\underline{x}) \leq m_{3}^{*}=223.781 \\
& v_{1^{*}}=0.519 \leq \hat{v}_{1}(\underline{x}) \leq v_{1}^{*}=271.965 \\
& v_{2^{*}}=0.009 \leq \hat{v}_{2}(\underline{x}) \leq v_{2}^{*}=3.612 \\
& v_{3^{*}}=2.741 \leq \hat{v}_{3}(\underline{x}) \leq v_{3}^{*}=207.585
\end{aligned}
$$

The strength data $y_{1}$, wear data $y_{2}$ and length data $y_{3}$ are the larger-is-better characteristics, the larger-is-better characteristics and the nominal-is-best characteristics with the target value 150 . We obtained the results of simultaneous optimization based on the proposed $P_{d}$ measure of Equation (13). Table 3 indicates that the optimal setting for the constraint that $\lambda=0.3$ is $x_{1}=-1.00$, $x_{2}=1.00$ and $x_{3}=-0.37$ which produces a predicted value of $P_{d}=0.62$, the value of desirability function for the estimated mean responses $\left(d_{1}=0.98, d_{2}=0.83\right.$, $d_{3}=0.98$ ), and the value of desirability function for the estimated variance responses $\left(d_{1}^{*}=0.72, d_{2}^{*}=0.17, d_{3}^{*}=\right.$ 1.00) for $y_{1}, y_{2}$ and $y_{3}$ respectively.

\section{Conclusion}

The combined-array approach allows one to provide separate estimates for the mean response and for the variance response. Accordingly, we can apply the primary goal of the Taguchi methodology which is to obtain a target condition on the mean while achieving the variance, or to minimize the variance while constraining the mean. In this study we proposed the simultaneous-optimization measure $P_{d}$ of multiple quality characteristics(or responses). The proposed concept of $P_{d}$ measure is minimizing the deviation of the mean response from the target value constraining the mean and variance responses. The $P_{d}$ measure is easy to apply, and permits the user to make subjective judgements on the importance (or desirability) of each response.

In this study we assume that the noise variables would be uniformly distributed over the region of interest of noise variables. It will be of interest to consider the case when the noise variables are not uniformly distributed over the region of interest of noise variables.

\section{Acknowledgments}

This study was supported (in part) by research funds from Chosun University, 2011. 


\section{References}

[1] G. Taguchi, "Introduction to quality engineering: designing quality into products and process", UNIPUB/Kraus International, White Plains, NY., 1986.

[2] G. Taguchi, "System of experimental designs: engineering methods to optimize quality and minimize cost”, UNIPUB/Kraus International, White Plains, NY., 1987.

[3] R. N. Kackar, "Off-line quality control, parameter design, and the Taguchi method", J. Qual. Technol., Vol. 17, pp. 176-209, 1985.

[4] W. J. Welch, T .K. Yu, S. M. Kang, and J. Sacks, "Computer experiments for quality control by parameter design”, J. Qual. Technol., Vol. 22, pp. 15-22, 1990.

[5] G. G. Vining and R. H. Myers, "Combining Taguchi and response surface philosophies: A dual response approach", J. Qual. Technol., Vol. 22, pp. 38-45, 1990.
[6] G. E. P. Box and S. P. Jones, "Designing products that are robust to the environment", Total Quality Management, Vol. 3, pp. 265-282, 1992.

[7] A. C. Shoemaker, K. L. Tsui, and C. F. J. Wu, "Economical experimentation methods for robust design", Technometrics, Vol. 33, pp. 415-427, 1991.

[8] R. H. Myers, A. I. Khuri, and G. G. Vining, "Response surface alternative to the Taguchi robust parameter design approach", Am. Stat., Vol. 46, pp. 131-139, 1992.

[9] A. I. Khuri and M. Conlon, "Simultaneous optimization of multiple responses represented by polynomial functions", Technometrics, Vol. 23, pp. 363375, 1981.

[10] D. S. Huang, "Regression and econometrics methods", John Wiley \& Sons, New York., p. 188, 1970.

[11] G. Derringer and R. Suich, "Simultaneous optimization of several response variables", J. Qual. Technol., Vol. 12, pp. 214-219, 1980. 